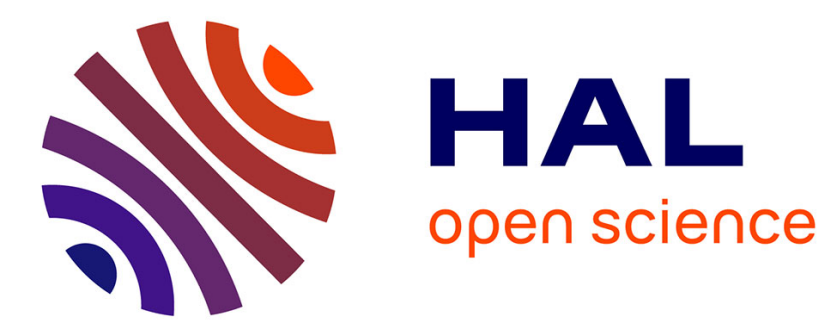

\title{
Symbiotic crystal-glass alloys via dynamic chemical partitioning
}

Ge Wu, Chang Liu, Andrea Brognara, Matteo Ghidelli, Yan Bao, Sida Liu, Xiaoxiang Wu, Wenzhen Xia, Huan Zhao, Jing Rao, et al.

\section{- To cite this version:}

Ge Wu, Chang Liu, Andrea Brognara, Matteo Ghidelli, Yan Bao, et al.. Symbiotic crystal-glass alloys via dynamic chemical partitioning. Materials Today, 2021, 51, pp.6-14. 10.1016/j.mattod.2021.10.025 . hal-03542403

\section{HAL Id: hal-03542403 https://hal.science/hal-03542403}

Submitted on 25 Jan 2022

HAL is a multi-disciplinary open access archive for the deposit and dissemination of scientific research documents, whether they are published or not. The documents may come from teaching and research institutions in France or abroad, or from public or private research centers.
L'archive ouverte pluridisciplinaire HAL, est destinée au dépôt et à la diffusion de documents scientifiques de niveau recherche, publiés ou non, émanant des établissements d'enseignement et de recherche français ou étrangers, des laboratoires publics ou privés. 


\section{Symbiotic crystal-glass alloys via dynamic chemical partitioning}

$\mathrm{Ge} \mathrm{Wu}^{1,} \uparrow$, Chang Liu ${ }^{1} \uparrow$, Andrea Brognara ${ }^{1}$, Matteo Ghidelli ${ }^{1,2, *}$, Yan Bao ${ }^{3}$, Sida Liu ${ }^{3}$, Xiaoxiang $\mathrm{Wu}^{4,1}$, Wenzhen Xia ${ }^{5,1}$, Huan Zhao ${ }^{1}$, Jing Rao ${ }^{1}$, Dirk Ponge ${ }^{1}$, Vivek Devulapalli ${ }^{1}$, Wenjun $\mathrm{Lu}^{6}$, ${ }^{1, *}$, Gerhard Dehm ${ }^{1}$, Dierk Raabe ${ }^{1, *}$, Zhiming $\mathrm{Li}^{1,7,8, *}$

1. Max-Planck-Institut für Eisenforschung, Max-Planck-Straße 1, 40237 Düsseldorf, Germany

2. Laboratoire des Sciences des Procédés et des Matériaux (LSPM), CNRS, Université Sorbonne Paris Nord, 93430, Villetaneuse, France

3. Department of Mechanical Engineering, City University of Hong Kong, Hong Kong, China

4. Shagang School of Iron and Steel, Soochow University, Suzhou 215137, China

5. School of Metallurgical Engineering, Anhui University of Technology, Maanshan, 243000 China

6. Department of Mechanical and Energy Engineering, Southern University of Science and Technology, Shenzhen, 518055, China

7. School of Materials Science and Engineering, Central South University, Changsha, 410083, China

8. State Key Laboratory of Powder Metallurgy, Central South University, 410083 Changsha, China

*Correspondence to: M. Ghidelli (Matteo.Ghidelli@1spm.cnrs.fr),W. Lu (luwj@ sustech.edu.cn), D. Raabe (d.raabe@mpie.de) or Z. Li (lizhiming@ csu.edu.cn).

$\dagger \mathrm{G}$. Wu and C. Liu contributed equally to this work

The design of high performance structural materials is always pursuing combinations of excellent yet often mutually exclusive mechanical properties and thermal stability. Although crystal-glass composite alloys provide better ductility compared to fully amorphous alloys, their thermal stability is poor, due to heterogeneous nucleation at the crystal-glass interface. Here we present a new strategy to develop thermally stable, ultrastrong and deformable crystal-glass nanocomposites through a thermodynamically guided alloy design approach, which mimics the mutual stabilization principle known from symbiotic ecosystems. We realized this in form of a model Cr-Co-Ni (crystalline)/Ti-Zr-Nb-Hf (amorphous) laminate 
composite alloy. The symbiotic alloy has an ultrahigh compressive yield strength of 3.6 GPa and large homogeneous deformation of $\sim 15 \%$ strain at ambient temperature, values which surpass those of conventional metallic glasses and nanolaminate alloys. Furthermore, the alloy exhibits $\sim 200 \mathrm{~K}$ higher crystallization temperature $\left(T_{X}>973 \mathrm{~K}\right)$ compared to that of the original TiZrNbHf-based amorphous phase. The elemental partitioning among adjacent amorphous and crystalline phases leads to their mutual thermodynamic and mechanical stabilization, opening up a new symbiotic approach for stable, strong and ductile materials.

Keywords: Crystal-glass composite alloys; Nanostructure; Thermal stability; Strong and ductile; Dynamic chemical partitioning

"Smaller is stronger" is an important characteristic of materials [1], which results from low probability of containing defects or defect sources in small sized samples. However, decreasing the size of materials (or materials' structural units) inevitably promotes heterogeneous nucleation $[2,3]$ from surfaces and interfaces at high temperatures, making them thermodynamically unstable, which leads to the long-standing trade-off dilemma between strength and thermal stability [4]. Nanocrystalline materials are stronger than their coarse-grain counterparts, yet experience rapid grain growth and loss of mechanical integrity at high temperatures [5]. Grain boundary segregation [6] or relaxation [7] can reduce the grain boundary energy of nanocrystalline materials, thus enhancing the thermal stability. For the ultrastrong amorphous materials, there has been a longstanding debate about the relation between the thermal stability and mechanical property of metallic glasses (MGs) concerning sample size. It was reported that the strength and deformability of MGs can be improved when reducing sample size [8-10], however, the thermal stability of the amorphous structure gets then usually weakened, due to heterogeneous nucleation [2,3] from surfaces. Similarly, the thermal stability of crystal-glass composite alloys is restricted by heterogeneous nucleation at the crystal-glass interface [11]. Here, we propose a novel alloy design concept for crystal-glass composites, which exploits annealing induced dynamic partitioning of elements from the crystal into the glassy phase to increase the crystallization temperature of the latter and enhance the deformability of the former, thus stabilizing the entire crystal-glass composite in a symbiotic fashion. We realized the symbiotic alloy concept in form of a crystalglass nanolaminate alloy, assisted by elemental partitioning among adjacent nanolayers during 
material preparation by sputter deposition. The partitioning enhances the negative mixing enthalpy of the recipient nanolayers, which promotes formation of the amorphous phase [12]. The donor nanolayers have a low glass-forming ability (GFA), thus forming a crystalline structure. Both, the crystalline and amorphous phases in the material show plastic necking and thinning features under tension and compression respectively, revealing their ductile nature. The further dynamic partitioning of elements from the crystal into the glassy phase during annealing enhances the thermal stability of the two symbiotic regions in the material.

We used magnetron sputtering to fabricate the crystal-glass symbiotic alloys by alternate deposition of $\sim 18 \mathrm{~nm}$-thick Cr-Co-Ni nanolayers and $\sim 12 \mathrm{~nm}$-thick Ti-Zr-Nb-Hf nanolayers. This architecture design is based on a topological confinement of the amorphous phase, which in turn enables its inelastic deformation. This effect is an important precondition for homogeneous plastic deformation of such composites [13,14]. Fig. 1 shows that the Cr-Co-Ni and Ti-Zr-Nb-Hf layers assume hexagonal-close-packed (HCP) and amorphous structures, respectively. This is significantly different from previous findings which showed that $\mathrm{CrCoNi}$ and TiZrNbHf alloys have face-centered-cubic (FCC) [15] and body-centered-cubic (BCC) [16] crystalline structures, respectively, in thermal equilibrium. The crystalline Cr-Co-Ni nanolayers in the current alloy grow with a strong $\left\{\begin{array}{llll}0 & 0 & 0 & 2\end{array}\right\}$ texture, as shown in the selected area electron diffraction (SAED) pattern in the inset of Fig. 1a. The CrCoNi alloy has a low stacking fault energy [17] of $22 \mathrm{~mJ}^{-2}$. Furthermore, Ni (31 at.\%), which is an FCC stabilizer, has a lower content in the crystalline phase than Co (33 at.\%) and $\mathrm{Cr}$ (36 at.\%). These aspects together with the non-equilibrium state generated by magnetron sputtering $\left(\sim 10^{10} \mathrm{~K} / \mathrm{s}\right.$ cooling rate) [18] promote HCP phase formation. Although TiZrNbHf is a typical BCC high-entropy alloy (HEA) [16], it forms an amorphous structure in the current material (Fig. 1c). The atom probe tomography (APT) results (Fig. 1e-g) show that the two kind nanolayers are mainly composed of CrCoNi and TiZrNbHf, respectively. However, the Ti-Zr-Nb-Hf nanolayers with a composition of $\mathrm{Ti}_{21.9} \mathrm{Zr}_{22.0} \mathrm{Nb}_{20.6} \mathrm{Hf}_{22.5} \mathrm{Cr}_{3.0} \mathrm{Co}_{2.5} \mathrm{Ni}_{7.5}$ (at.\%) contain certain amounts of $\mathrm{Cr}, \mathrm{Co}$ and $\mathrm{Ni}$ in the as-deposited sample, which were introduced by intermixing during the sputtering process [13]. According to one of Inoue's empirical laws for GFA [12], "a large negative mixing enthalpy" is a critical parameter for an enhanced GFA. Cr, Co and $\mathrm{Ni}$ have very large negative mixing enthalpy with $\mathrm{Ti}, \mathrm{Zr}, \mathrm{Nb}$ and $\mathrm{Hf}(<-23 \mathrm{KJ} / \mathrm{mol})$ [19,20], which promotes the GFA of the amorphous nanolayers. Similar phenomena have been observed 
in some TiZrHf-based metallic glasses (MGs), e.g. TiZrHfCuNi and TiZrHfCuCo [21]. After deposition of the amorphous phase, the subsequently sputtered Cr-Co-Ni layer has low GFA, hence forming a crystalline phase. The crystalline nanolayers have a lower surface diffusion rate compared to the amorphous nanolayers [22], which results in the negligible content of $\mathrm{Ti}, \mathrm{Zr}, \mathrm{Nb}$ and Hf within the Cr-Co-Ni nanolayers.
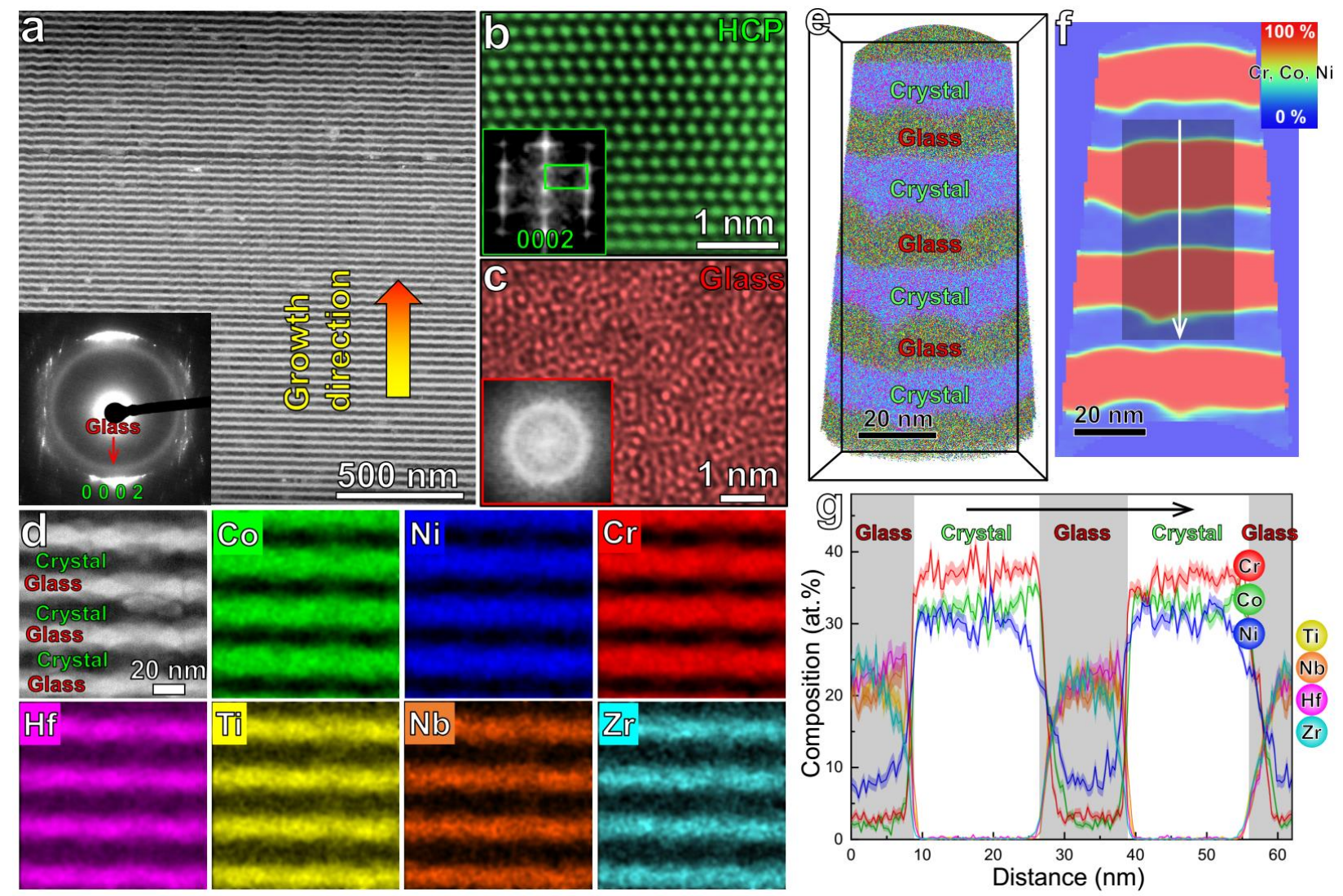

FIGURE 1. Microstructure and composition of the crystal-glass symbiotic alloy. a, Typical high angle annular dark-field (HAADF) scanning TEM (STEM) image. The inset is a typical SAED pattern obtained from side-view TEM probing, showing an amorphous ring and diffraction pattern with a strong $\left\{\begin{array}{llll}0 & 0 & 0 & 2\end{array}\right\}$ texture. $\mathbf{b}$ and $\mathbf{c}$, Side-view high-resolution (HR) STEM images showing HCP structure of the crystalline CrCoNi phase probed along the $<1 \quad 1-2$ 0 $>$ zone axis and mazelike pattern of the amorphous phase, respectively. The insets are corresponding fast Fourier transformation (FFT) images, showing that the $\left.\begin{array}{lllll}0 & 0 & 0 & 2\end{array}\right\}$ plane of the HCP phase is perpendicular to the growth direction and the glass phase presents a typical diffuse ring feature. d, HAADFSTEM and energy dispersion spectrum (EDS) mapping of the crystal-glass symbiotic alloy. e, 3D reconstruction of an APT dataset, showing the nano-laminated structure. f, 2D contour plot in terms of the $\mathrm{Cr}$, Co and $\mathrm{Ni}$ concentrations of a 1-nm-thick cross-sectional slice from the crystal- 
glass symbiotic alloy. g, 1D compositional profiles across the region indicated by the arrow in (f). The light shadows of the curves in (g) indicate statistical errors in terms of the standard deviations.

We next present the high thermal stability of the newly developed crystal-glass symbiotic alloy. The crystal-glass nanocomposite structure remains stable at $973 \mathrm{~K}$, as shown both, by in-situ TEM heating (Fig. 2a-c) and ex-situ annealing (Fig. 2e) experiments. After the ex-situ annealing experiments, TEM lamellae and APT tips were prepared from the annealed samples. The TiZrNbHf-based MGs [21] would be expected to crystallize under normal conditions at $\sim 773 \mathrm{~K}$ (Fig. S1). Here, however, we find a much higher thermal stability of the amorphous nanolayers in the current crystal-glass symbiotic alloy, revealing a $\sim 200 \mathrm{~K}$ higher $T_{X}$. To investigate the stabilization mechanism of the crystal-glass structure, chemical information of the crystal-glass symbiotic alloy after ex-situ annealing was retrieved using APT (Fig. 2f, g). It shows that the Ni and Co contents in the amorphous phase increase from 8 at.\% and 3 at.\% in the initial state to 37 at. $\%$ and 18 at. $\%$ after $623 \mathrm{~K}$ annealing for 1 hour, and to 37 at.\% and 30 at. $\%$ after $973 \mathrm{~K}$ annealing for 1 hour, respectively. This suggests that the TiZrNbHf-based amorphous nanolayers turn into a $\mathrm{Ni}$-enriched MG after annealing. Ni-based MGs are known to maintain their amorphous structure even at high temperatures $\left(T_{X}=\sim 940 \mathrm{~K}\right)$ [23]. We performed annealing at $623 \mathrm{~K}$ for 1 hour and 24 hours, respectively. Both annealed samples reveal HCP and amorphous nanolayers (Fig. S2) but did not form any Kirkendall voids [24]. This implies that the amorphous phase has sufficient sinks for vacancies, hence no voids are formed. Furthermore, the hardness of the current crystal-glass symbiotic alloy increases from the initial 6.4 GPa to $11 \mathrm{GPa}$ after annealing (Fig. 2d), also due to the enrichment of $\mathrm{Ni}$ and $\mathrm{Co}$ in the amorphous nanolayers. What is important here is that $\mathrm{Ni}$ and Co both have a large negative enthalpy of mixing with $\mathrm{Ti}, \mathrm{Zr}$, Nb and $\mathrm{Hf}(<-23 \mathrm{KJ} / \mathrm{mol})$ [19,20], thus stabilizing the amorphous structure and dynamically increasing $T_{X}$. Because the Young's modulus has a positive correlation [25] with $T_{X}$, MGs with higher $T_{X}$ are usually stronger when considering the $\sigma_{y}=E / 50$ relation [25] in various MG systems ( $\sigma_{y}$ is the yield strength, $E$ is the Young's modulus). Therefore, this dynamic elemental partitioning mechanism suppresses crystallization of the amorphous phase at high temperatures and further enhances the mechanical stiffness of the crystal-glass symbiotic composite. Although one might replace the TiZrNbHfbased MG nanolayers with Ni-Co-based MG nanolayers to achieve even higher hardness for the as-deposited sample, the smaller compositional differences between the Ni-Co-based MG and the 
crystalline Cr-Co-Ni nanolayers might reduce elemental partitioning. Dynamic partitioning during annealing has been rarely observed in crystal-glass nanolaminates with small compositional differences between the crystal and glass nanolayers, such as amorphous $\mathrm{CuZr}$-crystalline $\mathrm{Cu}$ system [26].

We note that the thickness of the crystalline nanolayers decreases from $\sim 18 \mathrm{~nm}$ to $\sim 17 \mathrm{~nm}$ (623 K, Fig. S2) and further to $\sim 15.5 \mathrm{~nm}$ (973 K, Fig. 2e) after annealing, which is caused by mass transfer ( $\mathrm{Ni}$ and $\mathrm{Co}$ ) from the crystalline phase to the adjacent amorphous phase. It is also important to note that the symbiotic effect acts in both directions, i.e. the partitioning effect also changes the adjacent HCP nanolayers: in some regions, the HCP crystal transforms into an FCC nano-structure after annealing at $973 \mathrm{~K}$ for 1 hour (Fig. 2e). Usually, HCP structures have limited ductility, due to the small number of independent slip systems [27]. Its partial transformation into FCC during annealing enhances its formability. Next, we discuss the deformation mechanisms of the symbiotic alloy.
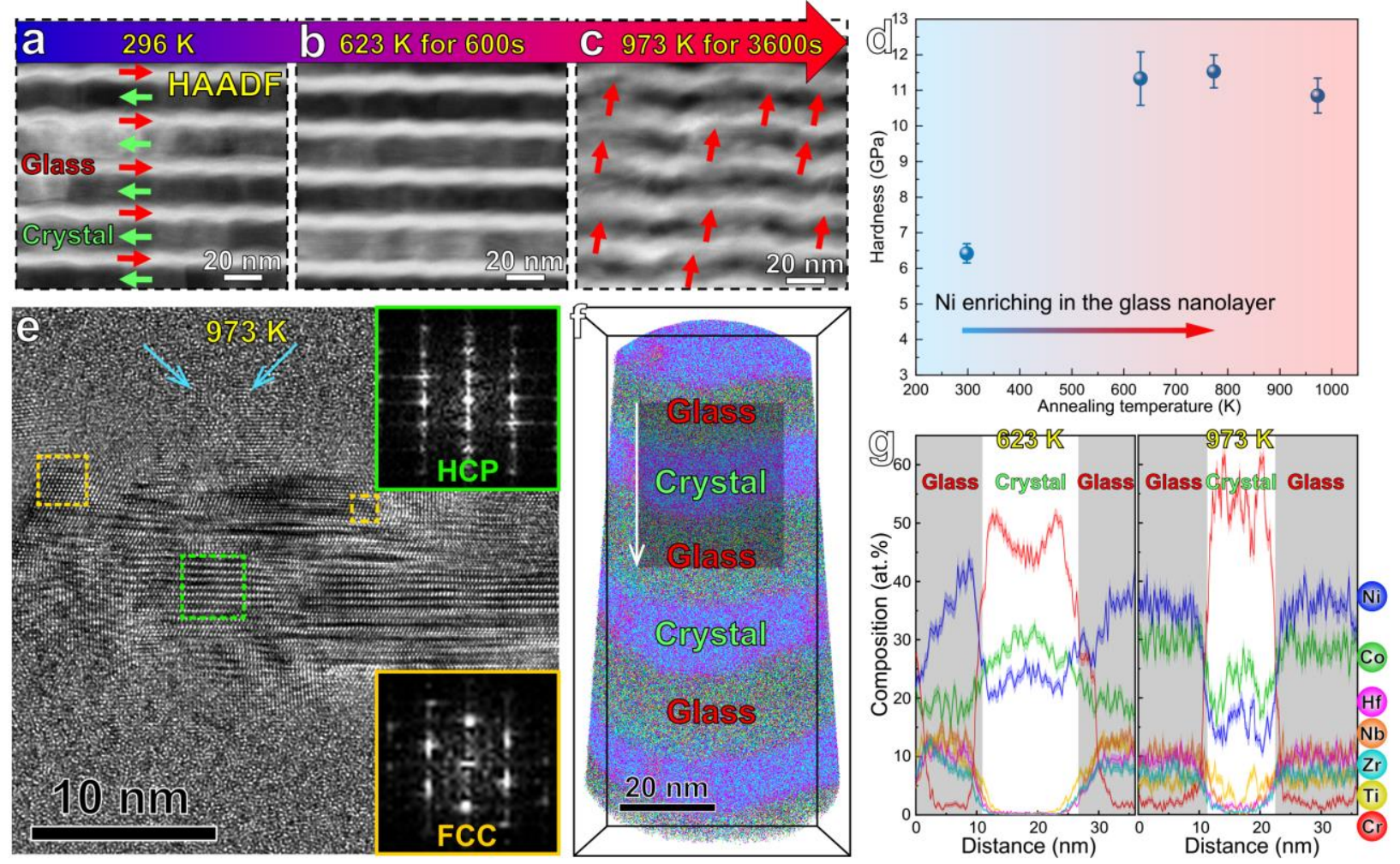

FIGURE 2. Thermal stability of the crystal-glass symbiotic alloy. HAADF-STEM images of the symbiotic alloy during in-situ heating at a, room temperature, b, $623 \mathrm{~K}$ annealing for $600 \mathrm{~s}$, c, 973 $\mathrm{K}$ annealing for $3600 \mathrm{~s}$. The red and green arrows in (a) denote the glass and crystal nanolayers, respectively. The red arrows in (c) indicate that the wavy-like feature of the symbiotic alloy 
becomes pronounced after $973 \mathrm{~K}$ annealing. d, Hardness of the symbiotic alloy after annealing at various high temperatures for 1 hour. The hardness increases as increasing the temperature from room temperature to $773 \mathrm{~K}$. The hardness decreases slightly when increasing temperature from $773 \mathrm{~K}$ to $973 \mathrm{~K}$, which may be due to the pronounced wavy-like feature of the crystal-glass nanocomposite structure. e, Aberration-corrected HRTEM image of the sample after ex-situ annealing at $973 \mathrm{~K}$ for 1 hour, showing the crystal-glass nanocomposite structure is sustained. The upper right and lower right insets are FFT images of the areas marked by dashed green and dashed yellow rectangles, indicating HCP and FCC structures, respectively. Some tiny crystals in the amorphous nanolayer near to the crystalline nanolayer (indicated by the arrows) may result from an incomplete amorphization after annealing, with a reduced GFA due to the depletion of $\mathrm{Ti}, \mathrm{Zr}$, $\mathrm{Nb}$ and $\mathrm{Hf}$ near the crystal-glass interface. $\mathbf{f}, 3 \mathrm{D}$ reconstruction of a typical APT dataset of the sample after annealing at $623 \mathrm{~K}$ for 1 hour. g, 1D compositional profiles across the region indicated by the arrow in (f) for the samples after annealing at $623 \mathrm{~K}$ and $973 \mathrm{~K}$ for 1 hour, respectively. The light shadows of the curves indicate statistical errors in terms of the standard deviations.

We conducted in-situ tension experiments in an aberration-corrected TEM to reveal the deformation behavior of the crystal-glass symbiotic alloy (as-deposited sample) at ambient temperature (Fig. 3). During tension, a crack is generated from the pre-notch of the TEM lamella and its propagation stops in front of an amorphous phase (Fig. 3b, c). The thickness of the crystalline phase ahead decreases from $\sim 18 \mathrm{~nm}$ to $\sim 14 \mathrm{~nm}$ as the tension process continues (Fig. $3 \mathrm{~d}_{1}-\mathrm{d}_{3}$ ), indicating a remarkable ductility. The lattice-resolved HRTEM image shows that the original HCP structure of the crystalline phase transforms to $\mathrm{HCP}+\mathrm{FCC}$ and at last fully to FCC during tensile loading (Fig. $3 \mathrm{f}_{1}-\mathrm{f}_{3}$ ). The $\frac{\mathrm{a}}{3}<1-1 \quad 0 \quad 0>$ partial dislocations gliding on every second $\left\{\begin{array}{llll}0 & 0 & 0 & 1\end{array}\right\}$ plane gradually convert HCP to FCC [28]. During the last stage of the tension test, strikingly, the failure mode of both the crystalline and amorphous phases is necking instead of shear deformation (Fig. 3g-i), emphasizing its ductile nature. Usually, alloys with HCP crystal structure, with their limited number of independent slip systems, have poor ductility [27]. However, the partial transformation into an FCC structure during tension adds further degrees of kinematic freedom in terms of the additional partial dislocations, an effect which enhances ductility [28]. Atomic strain mapping of a sample after tensile deformation shows that the FCC structure sustains higher shear strain compared to the HCP matrix (Fig. S3) in the crystalline phase, confirming the 
substantially higher plasticity of the FCC structure. Furthermore, the necking behavior of the MG phase is based on homogenous plastic flow, which is difficult to activate at ambient temperature in previously reported MGs [29]. For nanostructured MGs (such as nanoglasses [30]) with submicron sample size, deformability is enhanced compared to that of monolithic MGs, due to multiple shear banding modes [30], but not through homogeneous plastic flow as in the present case. For nanoglasses, it was proposed that the interfacial region with its reduced density (or enhanced excess free volume, respectively) serves as a preferential site for the initiation of embryonic shear bands, whose propagation can be hindered by the harder glassy region, generating multiple fine shear bands [30]. In contrast to this nanoglass deformation mechanism, the homogeneous plastic flow of the MG phase in the crystal-glass architecture can be activated in some cases where the volume fraction of the MG phase is low $(<20 \%)[13,14]$, i.e. the homogeneous plastic flow of the MG phase is confined by plastic deformation of the crystalline phase. Strikingly, the present symbiotic alloy has a high volume fraction of the MG phase ( 40\%) but still shows homogeneous plastic flow. The plastic flow of the MGs [8,9] at ambient temperature is usually attributed to the increased surface plasticity [31] when decreasing the sample size to sub-100 nm. In the current symbiotic alloy, the intrinsic small size of the MG phase $(\sim 12 \mathrm{~nm}$ thick $)$ and the strong and ductile property of the crystalline phase facilitate plastic flow while maintaining high strength. We note that it is difficult to locate dislocations in the fully deformed crystalline phase (Fig. S4). This suggests that the dislocations generated during deformation seem to get annihilated in the MG phase which acts as a dislocation sink [13,14,26] when undergoing plastic flow. The dislocation generation from the crystal-glass interfaces, their motion inside the crystalline phase, the shear-driven HCP to FCC phase transformation, and their annihilation at the opposite MG nanolayers provide the large ductility [14]. It is also important to note that the thickness of the crystalline phase decreases during plastic deformation. Therefore, the reduced dislocation mean-free-path due to the thinner crystalline phase and the associated higher frequency of internal interfaces, leads to an inversely increasing stress barrier [32] against dislocation motion, providing both, high strength and strain hardening. 


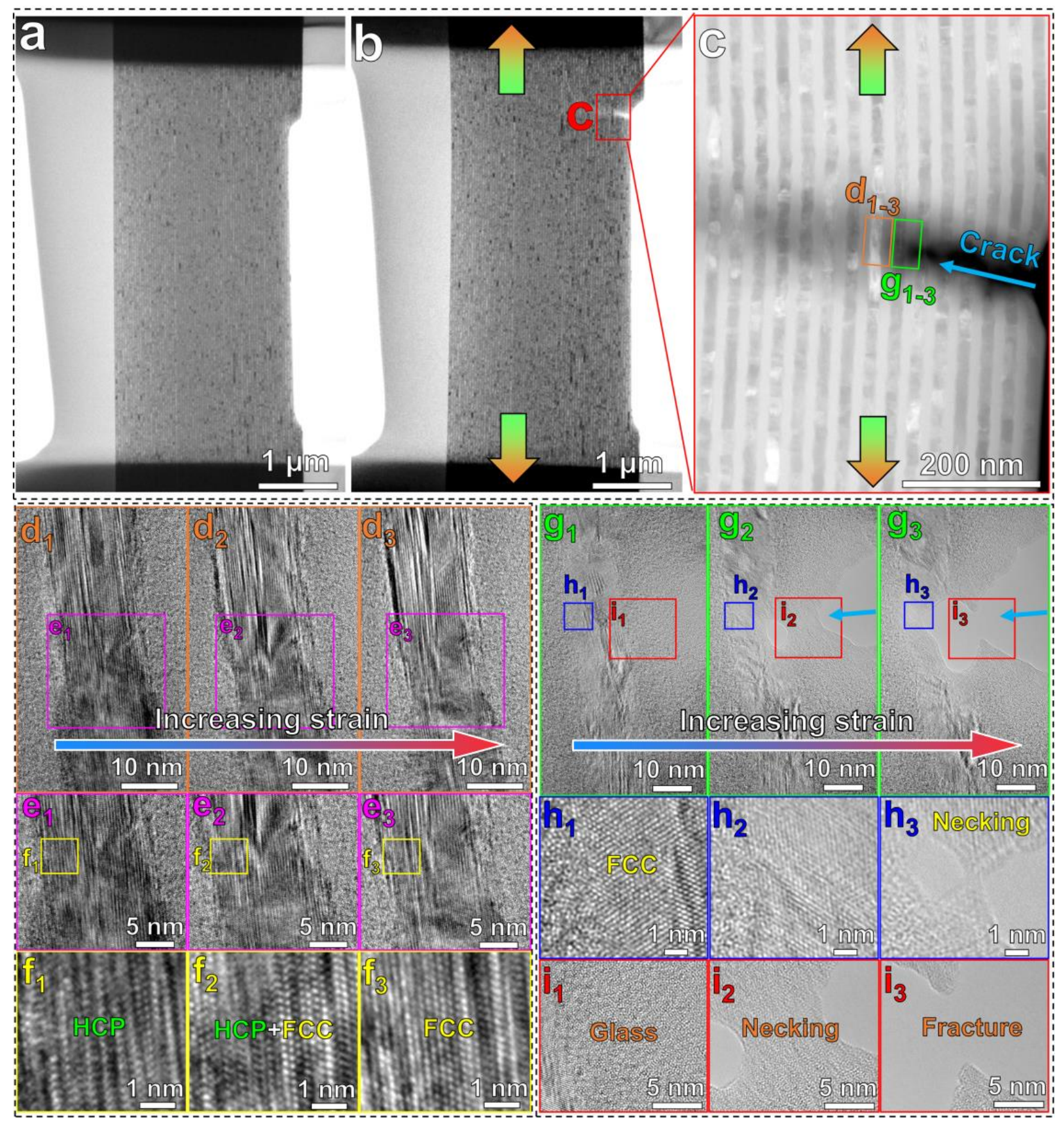

FIGURE 3. In-situ TEM tensile deformation behavior of the crystal-glass symbiotic alloy at room temperature. Bright-field TEM image of the crystal-glass symbiotic alloy $\mathbf{a}$, before and $\mathbf{b}$, during tension. c, Annular dark-field (ADF) STEM image of the enlarged area in (b), showing that a crack is generated during tension. $\mathbf{d}_{\mathbf{1}} \mathbf{-} \mathbf{d}_{\mathbf{3}}$, Snapshots of the HRTEM imaging of a crystalline nanolayer ahead of the crack during tension, showing that the thickness decreases from $\sim 18 \mathrm{~nm}$ to $\sim 14 \mathrm{~nm}$. e1-e3, Corresponding enlarged images of ( $\left.\mathbf{d}_{\mathbf{1}}-\mathbf{d}_{\mathbf{3}}\right)$. $\mathbf{f}_{\mathbf{1}}-\mathbf{f}_{\mathbf{3}}$, Corresponding lattice-resolved enlarged images of (e1-es), revealing the HCP to FCC phase transformation of the same area during tension. 
g1-g3, Snapshots of the HRTEM imaging of the crystalline and amorphous phases during fracture,

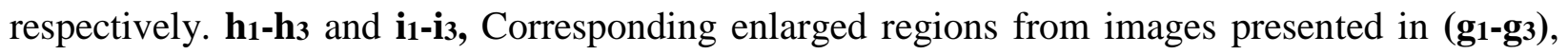
showing the necking feature of the crystalline and amorphous phases during fracture.

We also conducted microindentation on the surface of the symbiotic alloy at room temperature, and then investigated the structure and composition of the deformed material beneath the indent using aberration-corrected STEM and APT, respectively (Fig. S5). The deformed material shows no shear bands, different from deformed metallic glasses [33] and conventional crystallineamorphous nanolaminates [26], which form shear bands upon indentation. The thicknesses of the crystalline and amorphous nanolayers are both reduced (Fig. S5b-g), indicating co-deformation. The HCP to FCC phase transformation is also observed upon compressive deformation, identical to that observed in tension (Fig. 3). The compositions of the crystalline and amorphous phases are intermixed in the deformed material, indicating a mechanical alloying effect. The results can be interpreted in terms of a dislocation drag mechanism [26]: this means the dislocations generated in the crystalline phase drag $\mathrm{Ni}$ and Co towards the amorphous phase. The amorphous phase acts as a sink, absorbing the dislocations together with the dragged $\mathrm{Ni}$ and Co atoms.

The in-situ TEM tension (Fig. 3) and microindentation (Fig. S5) experiments reveal ductile deformation features of the crystal and glass phases in the symbiotic alloy, explaining the excellent mechanical response of the nanocomposite observed in the micro/nanopillar compression tests (with the layers perpendicular to the compression axis, Fig. 4). For comparison, we also performed identical tests on the partition-free reference materials, i.e. on nanocrystalline $\mathrm{CrCoNi}$ and TiZrNbHf-based $\left(\mathrm{Ti}_{19.0} \mathrm{Zr}_{19.3} \mathrm{Nb}_{19.0} \mathrm{Hf}_{19.3} \mathrm{Cr}_{8.1} \mathrm{Co} 7.7 \mathrm{Ni}_{7.6}\right.$ (at. \%)) amorphous alloys, which were produced using magnetron sputtering as well. It is worth noting that the mechanical properties of the reference TiZrNbHf-based amorphous alloy and the amorphous phase in the current symbiotic alloy are comparable, due to their similar composition [25]. The TiZrNbHf-based amorphous alloy has a yield strength of $1.8 \mathrm{GPa}$ (Fig. 4a), and fails soon after yielding, depicting a typical brittle signature of MGs. The serrations on the stress-strain curve correspond to the generation and propagation of shear bands (Fig. S6). The nanocrystalline CrCoNi alloy has a yield strength of 3.3 GPa and the engineering flow stress does not change substantially during plastic deformation (Fig. 4a), which usually indicates softening in compression. As the grain size of the nanocrystalline CrCoNi alloy is $\sim 20 \mathrm{~nm}$, the strain localization mechanisms of the nanocrystalline materials [34] 
account for softening during plastic deformation. As a consequence, the deformed sample shows shear deformation features (Fig. S6). Interestingly, the crystal-glass symbiotic alloy shows a much higher yield strength of $3.6 \mathrm{GPa}$ and homogeneous deformation of $\sim 15 \%$. The two reference alloys have a yield strength of $3.3 \mathrm{GPa}$ and $1.8 \mathrm{GPa}$, respectively, thus suggesting a linearly weighted average yield strength of only 2.7 GPa for the crystal-glass symbiotic alloy. Moreover, even if we assume that the yield strength of the amorphous phase in the symbiotic alloy approached the theoretical strength [9] of $\sim 3.1 \mathrm{GPa}$ due to the intrinsic size effect (referring to the size of the atomistic structural units that determine the material's cohesion), the yield strength of the whole material would be $3.2 \mathrm{GPa}$, still $0.4 \mathrm{GPa}$ below that observed in our experiments. Compared to the grain boundary structure, the crystal-glass interface provides a higher stress barrier against dislocation generation in the crystalline phase [14,35], which may induce the extra strengthening of $0.4 \mathrm{GPa}$. Furthermore, the symbiotic alloy undergoes $\sim 15 \%$ homogeneous deformation, due to the co-deformation of the crystal and glass phases. We note that no shear band is generated in the deformed sample (Fig. S7a), further confirming the homogeneous co-deformation of the two phases. We calculated the normalized strength $\left(\tau_{y} / G, \tau_{y}\right.$ and $G$ are shear yield strength and shear modulus, respectively) vs. homogeneous deformation relation of the crystal-glass symbiotic alloy, and compared it with that of conventional MGs and nanolaminate alloys (Fig. 4b). The length of the pillar samples is about or above $2 \mu \mathrm{m}$, i.e. two orders of magnitude above the nanolayer thickness, hence, the average material response is probed. The crystal-glass symbiotic alloy has a normalized shear strength to shear modulus ratio of $32 \times 10^{-3}$, near the theoretical strength limit [36] $\left(\sim 100 \times 10^{-3}\right)$. The homogeneous deformation regime is followed by a stress plateau and two larger stress drops (Fig. 4a, red arrows) due to cracking, although the pillar sample can be deformed to strains larger than 50\%. The cracks occur perpendicular to the layers (Fig. S7a). The crack generation can be impeded when decreasing the pillar diameter to $580 \mathrm{~nm}$ and $200 \mathrm{~nm}$, revealing a larger homogeneous strain of $\sim 20 \%$ and $>60 \%$, respectively. The deformation mechanism of the crystal-glass symbiotic alloy under compression is dislocation plasticity and HCP to FCC phase transformation in the crystalline phase and homogeneous plastic flow in the amorphous phase (Fig. S8), identical to the mechanisms observed under tension (Fig. 3). 


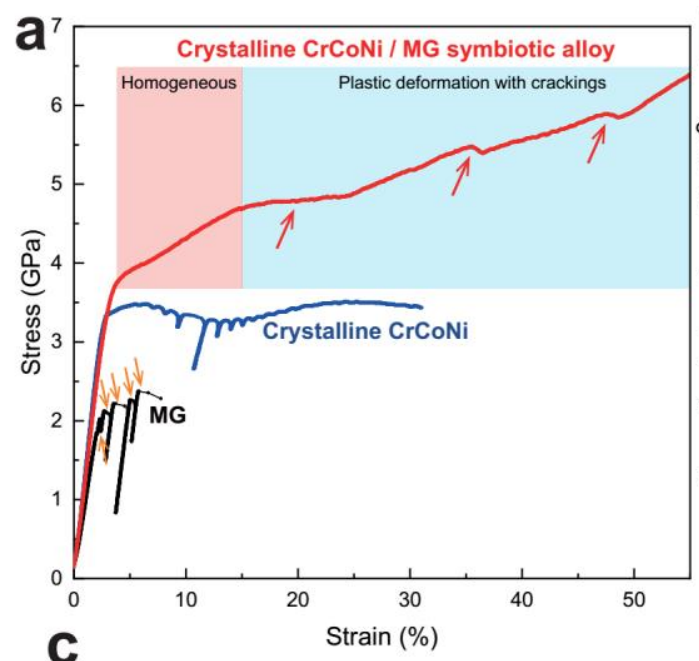

C

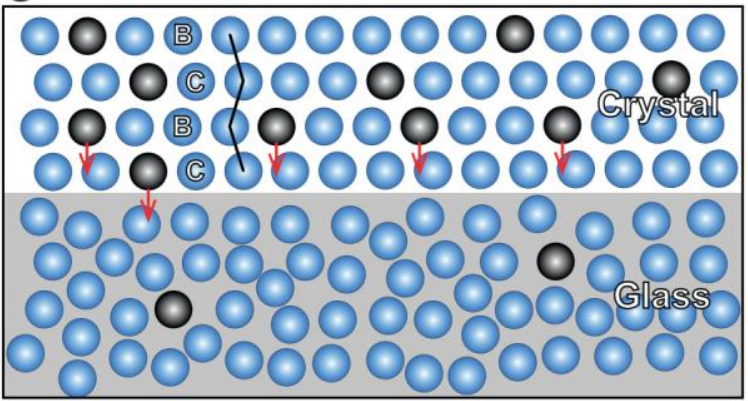

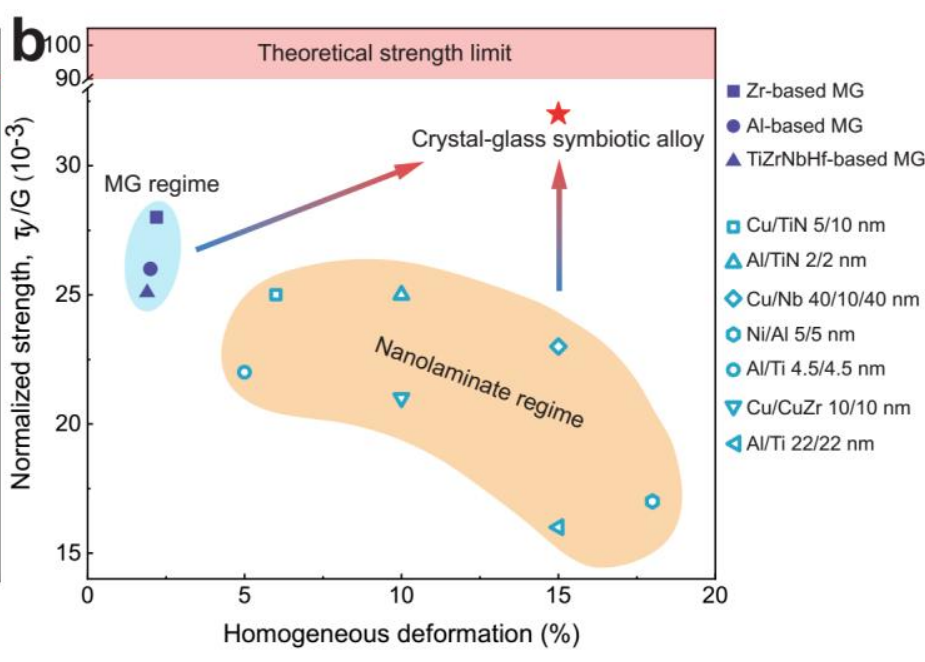

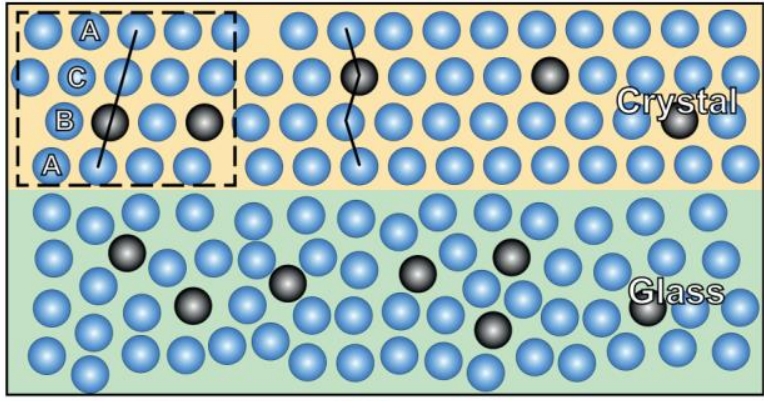

Heating
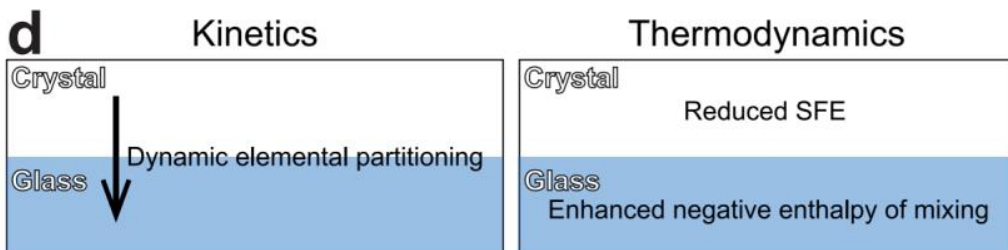

FIGURE 4. Mechanical properties of the crystal-glass symbiotic alloy. a, Compressive engineering stress-strain curves of the pillar samples with similar dimensions. The height/diameter ratio is 2 . The diameter is $1.2 \mu \mathrm{m}$ for the symbiotic alloy and $1.1 \mu \mathrm{m}$ for the crystalline $\mathrm{CrCoNi}$ and TiZrNbHf-based MG samples, respectively. The serration phenomenon in the MG sample is indicated by orange arrows. The cracking induced stress plateau and stress decrease for the symbiotic alloy are indicated by red arrows. b, Shear yield strength normalized by shear modulus vs. homogeneous deformation of the symbiotic alloy, tested in compression, in comparison with conventional MGs and nanolaminate alloys. The shear modulus $G$ is estimated using the equation $G=E /[2(1+v)]$, with $v$ being the Poisson's ratio and $E$ the Young's modulus, and $E=145 \mathrm{GPa}$ and $92 \mathrm{GPa}$ for the symbiotic alloy and TiZrNbHf-based MG, respectively, as obtained from nanoindentation. The shear yield strength $\tau_{y}$ is calculated using [37] $\tau_{y}=\sigma_{y} / 2$, where $\sigma_{y}$ is the yield 
strength. The source of the other data points are presented in Methods subsection 'Mechanical characterization'. c, Illustration of the alloy design strategy behind the symbiotic effects. The crystal and glass phases in the as-prepared alloy are in HCP (BCBC...sequence of the closest packed lattice plane) and amorphous state, respectively. During heating, dynamic elemental partitioning from the crystal into the glass phase (arrows in the left panel) enhances the negative mixing enthalpy of the glass phase, stabilizing the whole crystal-glass structure. The black and blue spheres represent atoms that partition more (e.g. Ni and $\mathrm{Co}$ ) or less (e.g. Ti, Zr, $\mathrm{Nb}$ and $\mathrm{Hf}$ ), respectively. The background of the glass phase is light black at the beginning (left panel) but becomes light green (right panel) after heat treatment, indicating an enhanced negative mixing enthalpy. A part of the HCP structure in the crystal phase transforms into an FCC structure (ACBA... sequence of the closest packed lattice planes) after heat treatment (dashed rectangle area in the right panel), due to a changed stacking fault energy (SFE) after the symbiotic partitioning. The background of the crystalline phase becomes light orange (right panel) after heat treatment, indicating the changed SFE. d, Schematic presentation of the three symbiotic effects: dynamic elemental partitioning from the crystal into the glass phase (kinetics); reduced stacking fault energy (SFE) of the crystalline phase and enhanced negative mixing enthalpy of the glass phase (thermodynamics); enhanced thermal stability of the crystal-glass structure, higher deformability of the crystalline phase by HCP to FCC phase transformation and homogeneous plastic flow of the glass phase (properties).

In summary, a novel crystal-glass symbiotic alloy with high thermal stability, ultrahigh strength and large plastic deformability was developed through a thermodynamically guided alloy design approach. It exploits mutual elemental partitioning among two adjacent phases to modify their individual properties in a way to enable their strengthening and compatible co-deformation, establishing a synergetic effect coined as 'symbiotic'. More specific, the concept was realized by dynamic partitioning of $\mathrm{Ni}$ and $\mathrm{Co}$ from the crystalline $\mathrm{Cr}-\mathrm{Co}-\mathrm{Ni}$ phase to the amorphous $\mathrm{Ti}-\mathrm{Zr}$ $\mathrm{Nb}$-Hf phase. The negative mixing enthalpy of the latter is enhanced while the former becomes more ductile due to partial HCP to FCC phase transformation. The dynamic elemental partitioning mechanism facilitates a stable crystal-glass structure up to $973 \mathrm{~K}$, i.e. $200 \mathrm{~K}$ higher than that for the original TiZrNbHf-based amorphous phase. The material exhibits a yield strength of 3.6 GPa and $\sim 15 \%$ homogeneous deformation at ambient temperature. The combination of ultrahigh 
strength and high deformability results from synergistic strengthening and ductilization of the crystal-glass nanocomposite structure, including HCP to FCC phase transformation of the crystalline phase, and theoretical strength and homogeneous plastic flow of the amorphous phase. These findings illustrate the advantages of the symbiotic mechanism in the multi-component crystal-glass nanocomposite structure (Fig. 4c, d). It is shown that this alloy- and nanostructure design approach enables materials with both, ultrahigh strength and large deformability and also with outstanding thermal stability. This combination makes symbiotic alloys attractive for applications in mechanically and thermally highly loaded environments, such as micro-electromechanical systems and flexible devices exposed to harsh thermal conditions.

\section{Methods}

Fabrication of the materials. We used magnetron sputtering as the fabrication method. The background vacuum was $4 \times 10^{-8}$ mbar. $\mathrm{CrCoNi}$ and TiZrNbHf alloy targets ( 99.9 at. $\%$ purity) were used for alternate sputtering. During sputtering, the target shutter was closed for $10 \mathrm{~s}$ after one target sputtering was finished, and then open the other target's shutter for deposition of another layer. The Ar working pressure was $4 \times 10^{-3}$ mbar, the temperature of the substrate was below 50 ${ }^{\circ} \mathrm{C}$, and the deposition rate of the $\mathrm{CrCoNi}$ and $\mathrm{TiZrNbHf}$ layers was $3 \mathrm{~nm} / \mathrm{min}$ and $5 \mathrm{~nm} / \mathrm{min}$, respectively. The samples with a thickness of $\sim 3 \mu \mathrm{m}$ and $\sim 2 \mu \mathrm{m}$ were deposited on $\mathrm{Si}\left(\begin{array}{lll}1 & 0 & 0\end{array}\right)$ and bulk $\mathrm{Fe}_{34} \mathrm{Ni}_{11} \mathrm{Mn}_{15} \mathrm{Co}_{20} \mathrm{Cr}_{20}$ (at.\%) HEA substrates, respectively. Nanocrystalline CrCoNi sample with a thickness of $\sim 3 \mu \mathrm{m}$ was fabricated with the same magnetron sputtering method using a CrCoNi targets (99.9\% purity). The amorphous $\mathrm{Ti}_{19.0} \mathrm{Zr}_{19.3} \mathrm{Nb}_{19.0} \mathrm{Hf}_{19.3} \mathrm{Cr}_{8.1} \mathrm{Co}{ }_{7.7} \mathrm{Ni}_{7.6}$ (at. \%) sample with a thickness of $\sim 2 \mu \mathrm{m}$ was fabricated by co-sputtering the $\mathrm{CrCoNi}$ and $\mathrm{TiZrNbHf}$ alloy targets (99.9 at.\% purity). The thickness of the films is large enough to fabricate micro-pillar samples by using a focused ion beam (FIB) facility.

Structural and compositional characterization. The microstructure of the crystal-glass symbiotic alloys was investigated by using (S)TEM. The cross-sectional TEM lamellae were prepared with a dual-beam FIB instrument (FEI Helios Nanolab 600i). The final milling voltage/current was $2 \mathrm{kV} / 23 \mathrm{pA}$, which was sufficiently small to reduce the FIB damage. We used a JEM 2200FS FEG transmission electron microscope (from JEOL) and image aberrationcorrected FEI Titan (Themis 80-300), operated at $200 \mathrm{kV}$ and $300 \mathrm{kV}$, respectively, to analyze TEM samples by bright-field imaging and electron diffraction. HR-STEM imaging and EDS were 
carried out using a $300 \mathrm{kV}$ probe aberration-corrected FEI Titan Themis. For high angle annular dark-field (HAADF) imaging a probe semi-convergence angle of $17 \mathrm{mrad}$ and inner and outer semi-collection angles ranging from 73 to 200 mrad were used. For ADF-STEM imaging, a probe semi-convergence angle of $17 \mathrm{mrad}$ and inner and outer semi-collection angles from 33 to $63 \mathrm{mrad}$ were used. For ABF-STEM imaging, a probe semi-convergence angle of $17 \mathrm{mrad}$ and inner and outer semi-collection angles from 13 to $21 \mathrm{mrad}$ were used. Needle-shaped specimens required for APT were fabricated by lift-outs and annular milled by FIB. The APT measurements were performed in a local electrode atom probe (CAMEACA LEAP 5000XR). The specimens were analyzed at $60 \mathrm{~K}$ in laser mode with a laser power of $20 \mathrm{pJ}$, a pulse repetition rate of $125 \mathrm{kHz}$, and an evaporation detection rate of $0.3 \%$ atom per pulse. Imago Visualization and Analysis Software (IVAS) version 3.8.4 was used for creating the 3D reconstructions and data analysis.

In-situ TEM tension and heating. The in-situ TEM tension at room temperature was conducted using a Gatan model 654 single-tilt straining holder via HRTEM mode with the normal e-beam density. The deformation behavior of the MG phase is not related to the e-beam illumination effect, based on the high crystallization temperature $\left(T_{X}>773 \mathrm{~K}\right)$ of the MG phase [21] in the current symbiotic alloy. The previous $80 \mathrm{~nm}$-thick $\mathrm{Cu}-\mathrm{Zr} \mathrm{MG}$ with much lower crystallization temperature $\left(T_{X}=\sim 723 \mathrm{~K}\right)$ reveal identical deformation behaviors under both the beam-on and beam-off conditions during in-situ TEM tensile testing [38]. The in-situ TEM heating was conducted using a Lighting heating/biasing holder from DENSsolutions (Delft, Netherlands) via TEM, STEM and EDS modes. A heating rate of approximately $100 \mathrm{~K} / \mathrm{s}$ was applied up to $1073 \mathrm{~K}$. All in-situ studies were carried out by means of an image aberration-corrected FEI Titan (Themis 80-300). The exsitu annealing experiment was conducted in a vacuum chamber with a base pressure of $10^{-6}$ mbar. Mechanical characterization. Micro-compression was performed using an Hysitron TI950 nanoindenter with a diamond punch, under displacement-control mode and at a strain rate of $\sim 5 \times 10^{-3} \mathrm{~s}^{-1}$. Micro-pillar samples were fabricated using FIB, with $30 \mathrm{kV} / 7 \mathrm{pA}$ as the final milling condition. The height of the pillars was maintained smaller than the film thickness. The aspect ratio (height/diameter) of the pillar was 2 , and the taper angle of each pillar was less than $1.5^{\circ}$. The engineering stress $\sigma$ was calculated using $F / A_{0}$, where $F$ is the measured force and $A_{0}$ is the original cross-sectional area at $20 \%$ of the pillar's height away from the top. The engineering strain $\varepsilon$ was calculated using $L / L_{0}$, where $L$ is the measured displacement and $L_{0}$ is the original length of the pillar. The mechanical properties of the current material were compared with those of conventional 
MGs [14,39] and nanolaminate alloys [40-45]. To perform the tensile testing experiments, flat bulk $\mathrm{Fe}_{34} \mathrm{Ni}_{11} \mathrm{Mn}_{15} \mathrm{Co}_{20} \mathrm{Cr}_{20}$ (at.\%) alloy substrates with a thickness of $1 \mathrm{~mm}$, gauge length of $4 \mathrm{~mm}$, and gauge width of $2 \mathrm{~mm}$ were sectioned from the as-homogenized alloy by electrical discharge machining. The crystal-glass symbiotic alloy films were then deposited on the surface of the alloy substrates. Uniaxial tensile tests were carried out at ambient temperature using a Kammrath \& Weiss tensile stage at a strain rate of $1 \times 10^{-3} \mathrm{~s}^{-1}$.

Data availability: All data are available in the manuscript or the supplementary materials.

Acknowledgments: Funding from the Deutsche Forschungsgemeinschaft (German Research Foundation, SPP 2006 and DE 796/9-2) is gratefully acknowledged. C.L. would like to acknowledge the financial support from European Union's Horizon 2020 Research and Innovation Programme (Grant Agreement 958457-FORGE). Z.L. would like to acknowledge the financial support from the National Natural Science Foundation of China (Grant No. 51971248).

Author contributions: G. Wu, Z. Li, M. Ghidelli and D. Raabe designed the project; G. Wu and C. Liu designed the material; G. Wu, A. Brognara and M. Ghidelli designed the magnetron sputtering experiment; G. Wu, C. Liu and D. Ponge conducted FIB and TEM experiments; G. Wu, C. Liu and H. Zhao conducted APT characterization and data analysis; X. Wu conducted arcmelting and ex-situ tension experiments; Y. Bao, S. Liu, W. Xia, J. Rao, C. Liu and G. Wu conducted microcompressions and microindentations; V. Devulapalli conducted ex-situ annealing experiments; W. Lu, G. Wu and G. Dehm conducted STEM characterization, in-situ tension and heating experiments; G. Wu, C. Liu, M. Ghidelli, W. Lu, Z. Li and D. Raabe wrote the paper; G. $\mathrm{Wu}$ and $\mathrm{C}$. Liu contributed equally to this work; all authors contributed to the discussion of the results.

Competing interests: The authors declare no competing interests.

\section{References}

1. J. R. Greer, and J. T. M. De Hosson, Prog. Mater. Sci. (2011) 56 (6), 654

2. W. Yan, et al., Nat. Nanotechnol. (2020), 1 
3. S. Sohn, et al., Nat. Commun. (2015) 6 (1), 1

4. M. Ames, et al., Acta Mater. (2008) 56 (16), 4255

5. P. Haasen, and B. L. Mordike, Physical Metallurgy. Cambridge Univ. Press, 1996:

6. T. Chookajorn, et al., Science (2012) 337 (6097), 951

7. X. Li, et al., Science (2020) 370 (6518), 831

8. H. Guo, et al., Nat. Mater. (2007) 6 (10), 735

9. D. Jang, and J. R. Greer, Nat. Mater. (2010) 9 (3), 215

10. M. Ghidelli, et al., Acta Mater. (2017) 131, 246

11. L.-C. Zhang, et al., J. Appl. Phys. (2006) 100 (3), 033514

12. A. Inoue, Acta Mater. (2000) 48 (1), 279

13. Y. Wang, et al., PNAS (2007) 104 (27), 11155

14. G. Wu, et al., Nat. Commun. (2019) 5099 (10), 1

15. B. Gludovatz, et al., Nat. Commun. (2016) 7 (1), 1

16. Z. Lei, et al., Nature (2018) 563 (7732), 546

17. G. Laplanche, et al., Acta Mater. (2017) 128, 292

18. P. Bordeenithikasem, et al., Sci. Rept. (2017) 7 (1), 1

19. S. Guo, et al., Intermetallics (2013) 41, 96

20. Y. Ye, et al., Mater. Today (2016) 19 (6), 349

21. L. Ma, et al., Mater. Trans. (2002) 43 (2), 277

22. C. Cao, et al., Appl. Phys. Lett. (2015) 107 (14), 141606

23. H. Choi-Yim, et al., Appl. Phys. Lett. (2003) 82 (7), 1030

24. E. Kirkendall, and A. Smigelskas, Aime Trans (1947) 171, 130

25. W. H. Wang, J. Appl. Phys. (2006) 99 (9), 093506

26. W. Guo, et al., Phys. Rev. Lett. (2014) 113 (3), 035501

27. M. Yoo, Metal. Trans. A (1981) 12 (3), 409

28. W. Lu, et al., Adv. Mater. (2018) 30 (44), 1804727

29. A. L. Greer, Science (1995) 267 (5206), 1947

30. X. L. Wang, et al., Scripta Mater. (2015) 98, 40

31. D. Z. Chen, et al., Nano Lett. (2013) 13 (9), 4462

32. X. Liu, et al., Science (2013) 342 (6156), 337

33. R. Bhowmick, et al., Acta Mater. (2006) 54 (16), 4221 
34. D. Jia, et al., Acta Mater. (2003) 51 (12), 3495

35. G. Wu, et al., Nature (2017) 545 (7652), 80

36. A. Kelly, and N. H. Macmillan, Oxford University Press, Walton Street, Oxford OX 26 DP, $U K$, 1986. (1986)

37. W. Johnson, and K. Samwer, Phys. Rev. Lett. (2005) 95 (19), 195501

38. L. Tian, et al., Acta Mater. (2013) 61 (13), 4823

39. J. Ye, et al., J. Appl. Phys. (2012) 112 (5), 053516

40. J. Zhang, et al., Acta Mater. (2012) 60 (20), 7183

41. Y. Zhang, et al., Scripta Mater. (2020) 186, 219

42. D. Bhattacharyya, et al., Acta Mater. (2011) 59 (10), 3804

43. R. Raghavan, et al., Mater. Sci. Eng. A (2015) 620, 375

44. Y. Chen, et al., Acta Mater. (2020) 199, 593

45. M. Nasim, et al., Acta Mater. (2020) 